\title{
Effect of charge renormalization on the electric and thermoelectric transport along the vortex lattice of a Weyl superconductor
}

\author{
G. Lemut, ${ }^{1}$ M. J. Pacholski, ${ }^{1}$ I. Adagideli, ${ }^{2}$ and C. W. J. Beenakker ${ }^{1}$ \\ ${ }^{1}$ Instituut-Lorentz, Universiteit Leiden, P.O. Box 9506, 2300 RA Leiden, The Netherlands \\ ${ }^{2}$ Faculty of Engineering and Natural Sciences, Sabanci University, Orhanli-Tuzla, 34956 Istanbul, Turkey
}

(Received 12 April 2019; published 15 July 2019)

\begin{abstract}
Building on the discovery that a Weyl superconductor in a magnetic field supports chiral Landau-level motion along the vortex lines, we investigate its transport properties out of equilibrium. We show that the vortex lattice carries an electric current $I=\frac{1}{2}\left(Q_{\text {eff }}^{2} / h\right)\left(\Phi / \Phi_{0}\right) V$ between two normal-metal contacts at voltage difference $V$, with $\Phi$ the magnetic flux through the system, $\Phi_{0}$ the superconducting flux quantum, and $Q_{\text {eff }}<e$ the renormalized charge of the Weyl fermions in the superconducting Landau level. Because the charge renormalization is energy dependent, a nonzero thermoelectric coefficient appears even in the absence of energy-dependent scattering processes.
\end{abstract}

DOI: 10.1103/PhysRevB.100.035417

\section{INTRODUCTION}

Weyl superconductors are nodal superconductors with topological protection [1,2]: they have nodal points of vanishing excitation gap, just like $d$-wave superconductors [3], but in contrast to those the gapless states are not restricted to high-symmetry points in the Brillouin zone and can appear for conventional $s$-wave pairing. The nodal points (Weyl points) at $\pm K$ in a Weyl superconductor are protected by the conservation of a topological invariant: the Berry flux of $\pm 2 \pi$ at Weyl points of opposite chirality $[4,5]$.

The distinction between symmetry and topology has a major consequence for the stability of Landau levels in a magnetic field. While in a $d$-wave superconductor the strong scattering of nodal fermions by vortices in the order parameter prevents the formation of Landau levels [6], in a Weyl superconductor an index theorem for chiral fermions protects the zeroth Landau level from broadening [7]. The appearance of chiral Landau levels in a superconducting vortex lattice produces a quantized thermal conductance parallel to the magnetic field, in units of $1 / 2$ times the thermal quantum per $h / 2 e$ vortex [7]. The factor of $1 / 2$ reminds us that Bogoliubov quasiparticles are Majorana fermions, "half a Dirac fermion" $[8,9]$.

In this paper we turn from thermal transport to electrical transport, by studying the geometry of Fig. 1 and addressing the question "What is the charge transported along the vortices in a chiral Landau level?" It is known [10] that the charge of Weyl fermions in a superconductor (pair potential $\Delta_{0}$ ) is reduced by a factor $\kappa=K\left(\Delta_{0}\right) / K(0)$. We find a direct manifestation of this charge renormalization in the electrical conductance, which is quantized at $\frac{1}{2}(e \kappa)^{2} / h$ per vortex. Because the charge renormalization is energy dependent, a coupling between thermal and electrical transport appears even without any energy-dependent scattering mechanism-resulting in a nonzero thermoelectric effect in a chiral Landau level.

In the next section, Sec. II, we summarize the effective low-energy theory of the superconducting vortex lattice [7], on which we base our scattering theory in Sec. III, followed by a calculation of electrical and thermoelectric transport properties in Sec. IV. These analytical results are compared with numerical simulations of a tight-binding model in Sec. V. We conclude in Sec. VI.

\section{LANDAU-LEVEL HAMILTONIAN IN THE VORTEX LATTICE}

We summarize the findings of Ref. [7] for the Landau-level Hamiltonian of Weyl fermions in a superconducting vortex lattice, for which we will need to calculate the transport properties.

\section{A. Dispersion relation}

A Landau level is a dispersionless flat band in the plane perpendicular to the magnetic field. The lowest (zeroth) Landau level is protected by chiral symmetry from scattering by the vortices; see Fig. 2. This is the Landau level on which we focus our analysis. It is a celebrated result of Nielsen and Ninomiya [4] that Weyl fermions in the zeroth Landau level have a definite chirality $\chi= \pm 1$, defined as the sign of the velocity $v_{z}=\partial E / \partial k_{z}$, parallel or antiparallel to $B$. To account for the electron-hole degree of freedom the number of bands is doubled for each chirality, so that we have four bands in total. Electronlike and holelike bands are related by the charge-conjugation symmetry relation $E_{\chi}\left(k_{z}\right)=-E_{\chi}\left(-k_{z}\right)$.

The effect of a superconducting vortex lattice on this fourband dispersion is given by [7]

$$
\begin{aligned}
& E_{\chi}\left(k_{z}\right)=-\left(\operatorname{sgn} k_{z}\right) \chi M\left(k_{z}\right)-\chi \mu \kappa\left(k_{z}\right), \\
& M\left(k_{z}\right)=\beta-\sqrt{\Delta_{0}^{2}+k_{z}^{2}}, \quad \kappa\left(k_{z}\right)=\frac{d}{d k_{z}} M\left(k_{z}\right),
\end{aligned}
$$

plotted in Fig. 3. (We have set $\hbar$ and the Fermi velocity $v_{\mathrm{F}}$ equal to unity, so $\kappa$ is dimensionless.) The magnitude of the superconducting pair potential outside of the vortex cores is denoted by $\Delta_{0}$ and $\beta$ is an internal magnetization along the 


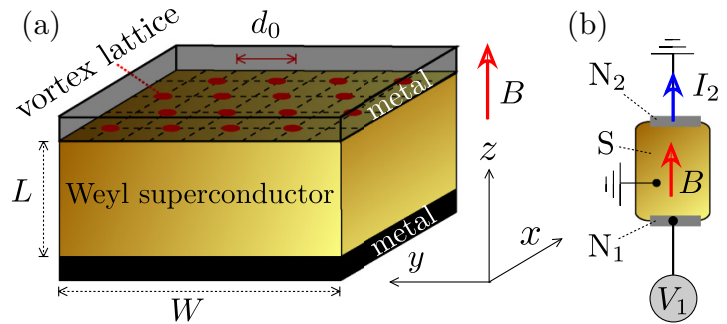

FIG. 1. (a) Vortex lattice in a Weyl superconductor sandwiched between metal electrodes; (b) circuit to measure the electrical transport along the vortex lines. The nonlocal conductance $G_{12}=d I_{2} / d V_{1}$ gives the current carried through the vortex lattice by nonequilibrium Weyl fermions in a chiral Landau level.

$z$ direction that breaks time-reversal symmetry even in the absence of any external magnetic field. In Eq. (2.1) we have assumed that $\beta$ is parallel to $B$, but we will later relax this assumption (see Sec. V C).

Provided that $\Delta_{0}<\beta$ there is a pair of Landau levels for each chirality, located in the magnetic Brillouin zone near the Weyl points at $k_{z}=K$ and $k_{z}=-K$, with [1]

$$
K\left(\Delta_{0}\right)=\sqrt{\beta^{2}-\Delta_{0}^{2}} .
$$

The charge expectation value

$$
Q_{\chi}=-e \frac{\partial E_{\chi}}{\partial \mu}=e \chi \kappa\left(k_{z}\right)=-\frac{e \chi k_{z}}{\sqrt{\Delta_{0}^{2}+k_{z}^{2}}}
$$

for a given chirality has the opposite sign at the two Weyl points. (We say that the chiral Landau levels near $k_{z}= \pm K$ are charge conjugate.) When $k_{z}= \pm K$ is at the Weyl point, the charge renormalization factor equals $\mp \kappa_{0}$, with

$$
\kappa_{0}=K\left(\Delta_{0}\right) / K(0)=\sqrt{1-\Delta_{0}^{2} / \beta^{2}}
$$

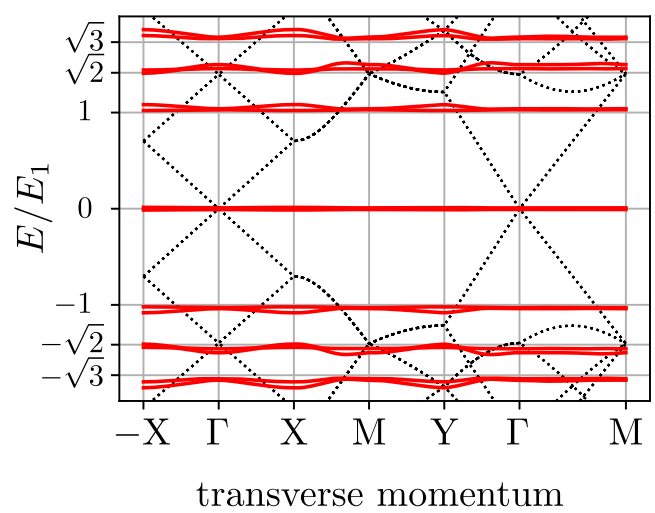

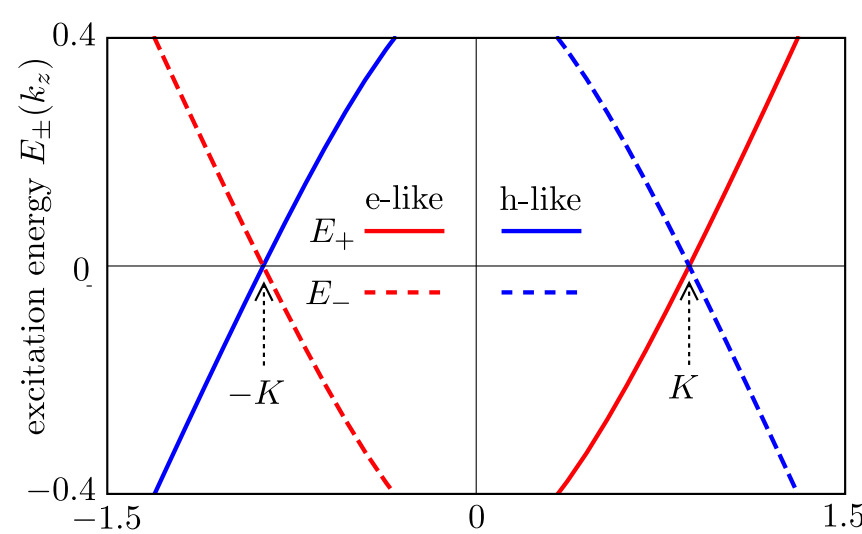

momentum $k_{z}$ parallel to the magnetic field

FIG. 3. Dispersion relation of the zeroth Landau level in a superconducting vortex lattice, plotted from Eq. (2.1) for $\mu=0, \Delta_{0}=0.5$, $\beta=1$. Only the dependence on the momentum $k_{z}$ along the magnetic field $B$ is shown; the dispersion is flat in the $x-y$ plane (see Fig. 2). The four branches are distinguished by the sign of the chirality (solid or dashed) and by the sign of the electric charge (red or blue). The zero-field Weyl points at $k_{z}= \pm K$ are indicated by arrows. Each branch has a degeneracy $N_{\text {Landau }}=e \Phi / h$ set by the enclosed flux $\Phi=B W^{2}$.

while $\kappa\left(k_{z}\right)$ varies linearly with energy away from the Weyl point [10].

\section{B. Effective Hamiltonian}

The dispersion (2.1) follows from the effective low-energy Hamiltonian [7],

$$
\mathcal{H}=U\left(\begin{array}{cccc}
H_{+} & 0 & 0 & 0 \\
0 & \cdot & \cdot & 0 \\
0 & . & \cdot & 0 \\
0 & 0 & 0 & H_{-}
\end{array}\right) U^{\dagger}
$$

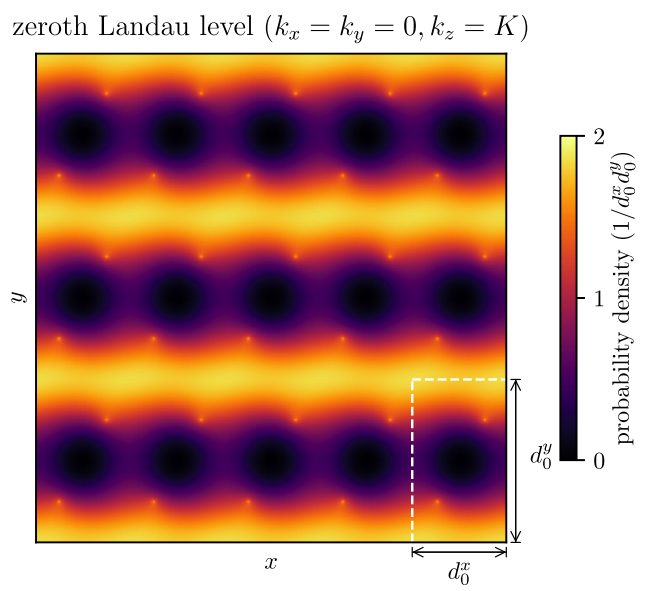

FIG. 2. Left: The red solid curves show the dispersion of Landau levels in the $k_{x}-k_{y}$ plane perpendicular to the magnetic field (energy $E$ normalized by the energy $E_{1}$ of the first Landau level). The black dotted curves show the dispersion in zero magnetic field, with a Weyl cone at the $\Gamma$ point of the magnetic Brillouin zone. Right: Particle density profile in the zeroth Landau level, in the $x-y$ plane perpendicular to the magnetic field, for a wave vector at the Weyl point $(\boldsymbol{k}=K \hat{z})$. The magnetic unit cell is indicated by a white dashed rectangle. Both panels are calculated numerically for a Weyl superconductor with a triangular vortex lattice. The vortex cores are located at the bright points in the density profile. Similar plots for a square vortex lattice are in Ref. [7]. 


$$
H_{\chi}=\left(k_{x}+e \mathcal{A}_{\chi, x}\right) \sigma_{x}+\left(k_{y}+e \mathcal{A}_{\chi, y}\right) \sigma_{y}+M \sigma_{z}-\chi \mu \kappa \sigma_{0},
$$

$$
U=\exp \left(\frac{1}{2} i \theta v_{y} \tau_{z} \sigma_{z}\right), \quad \theta=\arccos \kappa .
$$

The $2 \times 2$ Pauli matrices $v_{\alpha}, \tau_{\alpha}$, and $\sigma_{\alpha}$ (with $\alpha=0$ the corresponding unit matrix) act on, respectively, the electron-hole, orbital, and spin degrees of freedom. The full Hamiltonian $\mathcal{H}$ is an $8 \times 8$ matrix and the $2 \times 2$ matrices $H_{ \pm}$act on the $\sigma$ index in the $v=\tau= \pm 1$ sector.

The central block in Eq. (2.5a) indicated by dots refers to higher-lying bands that are approximately decoupled from the low-energy bands. Virtual transitions to these higher bands contribute order $\mu^{2}$ terms that remove the discontinuity in the derivative $\partial E / \partial k_{z}$ at $k_{z}=0$ for $\mu \neq 0$. No such decoupling approximations are made in the numerics of Sec. V.

The gauge field $\mathcal{A}_{\chi}(\boldsymbol{r})$, dependent on the position $\boldsymbol{r}=(x, y)$ in the $x-y$ plane, defines the effective magnetic field $\mathcal{B}_{\chi}=$ $\nabla \times \mathcal{A}_{\chi}$ in the $z$ direction felt by the Weyl fermions in the lattice of vortices at positions $\boldsymbol{R}_{n}$,

$$
\mathcal{B}_{\chi}=(1+\chi \kappa) \Phi_{0} \sum_{n} \delta\left(\boldsymbol{r}-\boldsymbol{R}_{n}\right)-\chi \kappa B .
$$

There are $N_{\text {vortex }}=B W^{2} / \Phi_{0}$ vortices of flux $\Phi_{0}=h / 2 e$ in an area $W^{2}$ perpendicular to the applied magnetic field $B$, so the spatial average $\int \mathcal{B}_{\chi} d \boldsymbol{r}=\Phi$ equals the total enclosed flux $\Phi=B W^{2}$ independent of $\kappa$ or of the lattice of vortices. (In the numerics that follows we will use a square lattice for definiteness.)

\section{Zeroth Landau-level wave functions}

As shown in Ref. [7], the Aharonov-Casher index theorem [11-13], together with the requirement that the wave functions are square-integrable at a vortex core, implies that the zeroth Landau-level eigenstates $\psi_{\chi}$ of $H_{\chi}$, which are rank-2 spinors, are also eigenstates $| \pm\rangle_{\sigma}$ of $\sigma_{z}$,

$$
\sigma_{z} \psi_{\chi}=\left(\operatorname{sgn} Q_{\chi}\right) \psi_{\chi} .
$$

The eigenvalue is determined by the sign of the effective quasiparticle charge (2.3).

It follows that the eigenstates $\Psi_{\chi}$ of the full Hamiltonian $\mathcal{H}$, which are rank- 8 spinors, have the form

$$
\begin{aligned}
\Psi_{\chi}= & e^{i k_{z} z} f_{\chi}(x, y) e^{(1 / 2) i \theta v_{y} \tau_{z} \sigma_{z}}|\operatorname{sgn} \chi\rangle_{\nu}|\operatorname{sgn} \chi\rangle_{\tau}\left|\operatorname{sgn} Q_{\chi}\right\rangle_{\sigma} \\
= & e^{i k_{z} z} f_{\chi}(x, y)\left[\cos (\theta / 2)|\operatorname{sgn} \chi\rangle_{\nu}|\operatorname{sgn} \chi\rangle_{\tau}\left|\operatorname{sgn} Q_{\chi}\right\rangle_{\sigma}\right. \\
& \left.-\sin (\theta / 2)\left(\operatorname{sgn} Q_{\chi}\right)|-\operatorname{sgn} \chi\rangle_{\nu}|\operatorname{sgn} \chi\rangle_{\tau}\left|\operatorname{sgn} Q_{\chi}\right\rangle_{\sigma}\right] .
\end{aligned}
$$

The spatial density profile $f_{\chi}(x, y)$ is peaked at the vortex cores, with a power-law decay $\left|f_{\chi}\right|^{2} \propto \delta r^{-1+\left|Q_{\chi}\right| / e}$ at a distance $\delta r$ from the core [7]. The renormalization of the quasiparticle charge does not affect the degeneracy of the zeroth Landau level: each of the four chiral modes in Fig. 3 has a degeneracy

$$
N_{\text {Landau }}=e \Phi / h
$$

set by the bare charge $e$.
Although the spatial density profile of these chiral modes is nonuniform, the wave functions extend over the entire $x-y$ plane-they are not exponentially confined to the vortex cores (see Fig. 2). This is a qualitative difference between the zeroth Landau level of a Weyl superconductor and zero modes bound to vortices in topological superconductors $[14,15]$.

\section{TRANSMISSION THROUGH THE NSN JUNCTION}

Referring to the geometry of Fig. 1, we seek the transmission matrix $t_{\mathrm{NSN}}$ for propagating modes of electrons and holes transmitted from the first metal contact $N_{1}$ in the region $z<0$, through the Weyl superconductor in the region $0<z<L$, into the second metal contact $N_{2}$ in the region $z>L$.

\section{A. Renormalized charge transfer}

We start by examining a single $N S$ interface, to study how a chiral mode in the superconductor injects a renormalized charge into the normal metal.

On the superconducting side $z<L$ of the $N S$ interface at $z=L$ the incident modes have positive chirality $\chi=+1$. There is a mode $\Psi_{\mathrm{S}}$ with perpendicular momentum $k_{z}$ near $K$ and a mode $\Psi_{\mathrm{S}}^{\prime}$ with $k_{z}^{\prime}$ near $-K$. We do not specify the transverse momentum $\boldsymbol{k}_{\|}=\left(k_{x}, k_{y}\right)$, which gives each mode a degeneracy of $N_{\text {Landau }}=e \Phi / h$; see Eq. (2.9).

According to Eq. (2.8), the spinor structure of the chiral modes is

$$
\begin{aligned}
& \Psi_{\mathrm{S}} \propto \cos (\theta / 2)|++-\rangle_{\nu \tau \sigma}+\sin (\theta / 2)|-+-\rangle_{\nu \tau \sigma}, \\
& \Psi_{\mathrm{S}}^{\prime} \propto \cos \left(\theta^{\prime} / 2\right)|+++\rangle_{\nu \tau \sigma}-\sin \left(\theta^{\prime} / 2\right)|-++\rangle_{\nu \tau \sigma} .
\end{aligned}
$$

We have abbreviated $| \pm \pm \pm\rangle_{\nu \tau \sigma}=| \pm\rangle_{\nu}| \pm\rangle_{\tau}| \pm\rangle_{\sigma}$ and denote $\theta=\theta\left(k_{z}\right), \theta^{\prime}=\theta\left(k_{z}^{\prime}\right)$.

For the normal metal we take the free-electron Hamiltonian

$$
H_{\mathrm{N}}=\frac{1}{2 m}\left(k^{2}-k_{\mathrm{F}}^{2}\right) \nu_{z} \tau_{0} \sigma_{0},
$$

isotropic in the spin and valley degrees of freedom, in the high Fermi-momentum limit $k_{\mathrm{F}} l_{m} \rightarrow \infty$ when the effect of the magnetic field on the spectrum may be neglected $\left(l_{m}=\sqrt{\hbar / e B}\right.$ is the magnetic length).

Because of the large potential step experienced upon traversing the $N S$ interface, the perpendicular momentum $k_{z}$ is boosted to $+k_{\mathrm{F}}$ for the electron component of the state and to $-k_{\mathrm{F}}$ for the hole component. A state in $N$ moving away from the $N S$ interface of the form

$$
\begin{gathered}
\Psi_{\mathrm{N}} \propto e^{i k_{\mathrm{F}}(z-L)} \cos (\theta / 2)|++-\rangle_{\nu \tau \sigma} \\
\quad+e^{-i k_{\mathrm{F}}(z-L)} \sin (\theta / 2)|-+-\rangle_{\nu \tau \sigma}
\end{gathered}
$$

can be matched to the incident state $\Psi_{\mathrm{S}}$ in $S$, while the state

$$
\begin{gathered}
\Psi_{\mathrm{N}}^{\prime} \propto e^{i k_{\mathrm{F}}(z-L)} \cos \left(\theta^{\prime} / 2\right)|+++\rangle_{\nu \tau \sigma} \\
-e^{-i k_{\mathrm{F}}(z-L)} \sin \left(\theta^{\prime} / 2\right)|-++\rangle_{\nu \tau \sigma}
\end{gathered}
$$

can be matched to $\Psi_{\mathrm{s}}^{\prime}$.

The charge transferred through the interface when $\Psi_{\mathrm{S}} \mapsto \Psi_{\mathrm{N}}$ equals the renormalized charge from Eq. (2.3),

$$
Q_{\mathrm{N}}=\left\langle\Psi_{\mathrm{N}}\left|e v_{z}\right| \Psi_{\mathrm{N}}\right\rangle=e \cos \theta=e \kappa=\frac{-e k_{z}}{\sqrt{\Delta_{0}^{2}+k_{z}^{2}}},
$$


dependent on the perpendicular momentum $k_{z}$ in $S$, before the boost to $k_{\mathrm{F}}$ in $N$. When $k_{z}=K$, this gives

$$
Q_{\mathrm{N}}=-e \sqrt{1-\Delta_{0}^{2} / \beta^{2}}=-\kappa_{0} e \equiv-Q_{\mathrm{eff}} .
$$

This is for the transmission $\Psi_{\mathrm{S}} \mapsto \Psi_{\mathrm{N}}$. The other transmission $\Psi_{\mathrm{S}}^{\prime} \mapsto \Psi_{\mathrm{N}}^{\prime}$ transfers for $k_{z}^{\prime}=-K$ a charge $Q_{\mathrm{N}}^{\prime}=+Q_{\text {eff }}$.

Similarly, at the opposite $N S$ interface $z=0$ the chiral Landau level modes in $S$ moving away from the interface are matched to incoming states in $N$ of the form

$$
\begin{aligned}
& \Phi_{\mathrm{N}} \propto e^{i k_{\mathrm{F}} z} \cos (\theta / 2)|++-\rangle_{\nu \tau \sigma}+e^{-i k_{\mathrm{F}} z} \sin (\theta / 2)|-+-\rangle_{\nu \tau \sigma} \\
& \Phi_{\mathrm{N}}^{\prime} \propto e^{i k_{\mathrm{F}} z} \cos \left(\theta^{\prime} / 2\right)|+++\rangle_{\nu \tau \sigma}-e^{-i k_{\mathrm{F}} z} \sin \left(\theta^{\prime} / 2\right)|-++\rangle_{\nu \tau \sigma}
\end{aligned}
$$

\section{B. Transmission matrix}

At a given energy $E$ relative to the Fermi level the perpendicular momenta $k_{z}$ and $k_{z}^{\prime}$ of the chiral Landau levels in $S$ moving in the $+z$ direction are determined by the dispersion relation (2.1) with $\chi=+1$. For $\mu=0$ the expressions are simple,

$$
k_{z}=K+(\beta / K) E, \quad k_{z}^{\prime}=-K+(\beta / K) E .
$$

For any $\mu$, particle-hole symmetry ensures that

$$
k_{z}(E)=-k_{z}^{\prime}(-E) \text {. }
$$

The Landau level $\Psi_{\mathrm{S}}$ propagating from $z=0$ to $z=L$ accumulates a phase $k_{z} L$, and similarly $\Psi_{\mathrm{S}}^{\prime}$ accumulates a phase $k_{z}^{\prime} L$. The full transmission matrix of the $N S N$ junction at energy $E$ can thus be written as

$$
t_{\mathrm{NSN}}(E)=e^{i k_{z} L}\left|\Psi_{\mathrm{N}}\right\rangle\left\langle\Phi_{\mathrm{N}}\left|+e^{i k_{z}^{\prime} L}\right| \Psi_{\mathrm{N}}^{\prime}\right\rangle\left\langle\Phi_{\mathrm{N}}^{\prime}\right|,
$$

with $k_{z}$ and $k_{z}^{\prime}$ determined by Eq. (3.7).

We can rewrite Eq. (3.9) in the basis of propagating electron modes in the normal metal. In the region $z<0$ one has the basis states

$$
\begin{aligned}
& \left|\Psi_{\uparrow}\right\rangle=\left(\begin{array}{l}
|e \uparrow\rangle \\
|h \uparrow\rangle
\end{array}\right), \quad\left|\Psi_{\downarrow}\right\rangle=\left(\begin{array}{c}
|e \downarrow\rangle \\
|h \downarrow\rangle
\end{array}\right), \\
& |e \uparrow\rangle=e^{i k_{\mathrm{F}} z}|+++\rangle_{\nu \tau \sigma}, \quad|h \uparrow\rangle=e^{-i k_{\mathrm{F}} z}|-++\rangle_{\nu \tau \sigma}, \\
& |e \downarrow\rangle=e^{i k_{\mathrm{F}} z}|++-\rangle_{\nu \tau \sigma}, \quad|h \downarrow\rangle=e^{-i k_{\mathrm{F}} z}|-+-\rangle_{\nu \tau \sigma},
\end{aligned}
$$

and similarly for $z>L$ with $k_{\mathrm{F}} z$ replaced by $k_{\mathrm{F}}(z-L)$.

The transmission matrix is block diagonal in the spin degree of freedom,

$$
\begin{aligned}
t_{\mathrm{NSN}}(E) & =\left(\begin{array}{cc}
t_{\uparrow}(E) & 0 \\
0 & t_{\downarrow}(E)
\end{array}\right), \\
t_{\uparrow} & =e^{i k_{z}^{\prime} L}\left(\begin{array}{cc}
\cos ^{2}\left(\theta^{\prime} / 2\right) & -\cos \left(\theta^{\prime} / 2\right) \sin \left(\theta^{\prime} / 2\right) \\
-\cos \left(\theta^{\prime} / 2\right) \sin \left(\theta^{\prime} / 2\right) & \sin ^{2}\left(\theta^{\prime} / 2\right)
\end{array}\right), \\
t_{\downarrow} & =e^{i k_{z} L}\left(\begin{array}{cc}
\cos ^{2}(\theta / 2) & \cos (\theta / 2) \sin (\theta / 2) \\
\cos (\theta / 2) \sin (\theta / 2) & \sin ^{2}(\theta / 2)
\end{array}\right) .
\end{aligned}
$$

The $2 \times 2$ matrix $t_{\uparrow}$ acts on the electron-hole spinor $\left|\Psi_{\uparrow}\right\rangle$ and $t_{\downarrow}$ acts on $\left|\Psi_{\downarrow}\right\rangle$. We may write this more compactly as

$$
\begin{aligned}
& t_{\uparrow}=\frac{1}{2} e^{i k_{z}^{\prime} L}\left(v_{0}+v_{z} e^{-i \theta^{\prime} v_{y}}\right), \\
& t_{\downarrow}=\frac{1}{2} e^{i k_{z} L}\left(v_{0}+v_{z} e^{i \theta v_{y}}\right) .
\end{aligned}
$$

These are each rank-1 matrices; one eigenvalue equals 0 and the other equals 1 in absolute value. The unit transmission eigenvalue is $N_{\text {Landau-fold degenerate in the transverse mo- }}$ mentum $\boldsymbol{k}_{\|}$.

At the Fermi level $E=0$ the particle-hole symmetry relation (3.8) implies $k_{z}^{\prime}=-k_{z}, \theta^{\prime}=\pi-\theta$, hence

$$
t_{\mathrm{NSN}}(0)=\frac{1}{2} e^{-i k_{z} L \sigma_{z}}\left(v_{0}-v_{z} \sigma_{z} e^{i \theta v_{y}}\right)
$$

One verifies that

$$
t_{\mathrm{NSN}}(0)=v_{y} \sigma_{y} t_{\mathrm{NSN}}^{*}(0) v_{y} \sigma_{y},
$$

as required by particle-hole symmetry.

\section{TRANSPORT PROPERTIES}

The transmission matrix allows us to calculate the transport properties of the NSN junction, under the assumption that there is no backscattering of the chiral modes in the Weyl superconductor. To simplify the notation, we write $t$ for the Fermi-level transmission matrix $t_{\mathrm{NSN}}(0)$. The submatrices of electron and hole components are denoted by $t_{e e}, t_{h h}, t_{h e}$, and $t_{e h}$. We define the combinations

$$
\begin{aligned}
& \mathcal{T}_{ \pm}=t_{e e}^{\dagger} t_{e e} \pm t_{h e}^{\dagger} t_{h e}, \\
& \mathcal{T}_{+}=\frac{1}{2}\left(v_{0}+v_{z}\right) t^{\dagger} t, \quad \mathcal{T}_{-}=\frac{1}{2}\left(v_{0}+v_{z}\right) t^{\dagger} v_{z} t
\end{aligned}
$$

\section{A. Thermal conductance}

As a check, we first recover the result of Ref. [7] for the quantization of the thermal conductance.

The thermal conductance $G_{\text {thermal }}=J_{12} / \delta T$ gives the heat current $J_{12}$ transported at temperature $T_{0}$ from contact $N_{1}$ to $N_{2}$ via the superconductor, in response to a small temperature difference $\delta T$ between the contacts. It follows from the total transmitted quasiparticle current that

$$
G_{\text {thermal }}=\frac{1}{2} g_{0} N_{\text {Landau }} \operatorname{Tr} t^{\dagger} t=g_{0} \frac{e \Phi}{h},
$$

with $N_{\text {Landau }}=e \Phi / h$ the Landau-level degeneracy and $g_{0}=$ $\frac{1}{3}\left(\pi k_{\mathrm{B}}\right)^{2}\left(T_{0} / h\right)$ the thermal conductance quantum. The factor $1 / 2$ in the first equation appears because the quasiparticles 
in the Weyl superconductor are Majorana fermions. It is canceled by the factor of 2 from $\operatorname{Tr} t t^{\dagger}=2$, in view of Eq. (3.13).

\section{B. Electrical conductance}

Referring to the electrical circuit of Fig. 1(b), we consider the electrical conductance $G_{12}=d I_{2} / d V_{1}$, given by

$$
\begin{aligned}
G_{12} & =\frac{e^{2}}{h} N_{\text {Landau }} \operatorname{Tr} \mathcal{T}_{-} \\
& =\frac{e^{2}}{h} N_{\text {Landau }} \frac{1}{2} \operatorname{Tr}\left(v_{0}+v_{z}\right) t^{\dagger} v_{z} t .
\end{aligned}
$$

In the linear response limit $V_{1} \rightarrow 0$ we substitute $t$ from Eq. (3.13), which gives

$$
G_{12}(0)=\cos ^{2} \theta \frac{e^{2}}{h} N_{\text {Landau }}=\frac{(e \kappa)^{2}}{h} \frac{e \Phi}{h} .
$$

The conductance quantum $e^{2} / h$ is renormalized by the effective charge $e \mapsto e \kappa$. At $\mu=0$, when $k_{z}=K$, the renormalization factor is $\kappa_{0}^{2}=\left(Q_{\text {eff }} / e\right)^{2}=1-\Delta_{0}^{2} / \beta^{2}$ from Eq. (3.5). Note that the conductance per $h / 2 e$ vortex is $\frac{1}{2}\left(e \kappa_{0}\right)^{2} / h$, with an additional factor $1 / 2$ to signal the Majorana nature of the quasiparticles.

At finite $E=e V_{1}$ we must use the energy-dependent transmission matrix (3.11), which gives

$$
G_{12}(E)=\frac{1}{2} \frac{e^{2}}{h} N_{\text {Landau }}\left(\cos \theta+\cos \theta^{\prime}+\cos ^{2} \theta+\cos ^{2} \theta^{\prime}\right) .
$$

Substituting Eq. (3.4) for $\cos \theta$ and $\cos \theta^{\prime}$ at $k_{z}$ and $k_{z}^{\prime}$, given as a function of $E$ by Eq. (3.7), we find

$$
G_{12}(E)=G_{12}(0)\left(1-\frac{\Delta_{0}^{2} E}{\left(\beta^{2}-\Delta_{0}^{2}\right)^{3 / 2}}+\mathcal{O}\left(E^{2}\right)\right) .
$$

The energy dependence of the differential conductance comes entirely from the energy dependence of the effective charge: At $E=0$ the electronlike and holelike chiral Landau levels have precisely opposite effective charge $\pm Q_{\text {eff }}$, but for $E \neq 0$ the effective charges differ in absolute value by an amount $\propto d k_{z} / d E$.

\section{Shot noise}

At temperatures small compared to the applied voltage $V_{2}$, the time-dependent fluctuations in the current $I_{2}$ are due to shot noise. The formula for the shot-noise power is [16]

$$
P_{12}=\frac{e^{3} V_{1}}{h} \operatorname{Tr}\left(\mathcal{T}_{+}-\mathcal{T}_{-}^{2}\right) .
$$

This can again be written in terms of the Pauli matrix $\tau_{z}$ and evaluated using Eq. (3.13),

$$
P_{12}=\frac{e^{3} V_{1}}{h}\left(1-\frac{1}{2} \kappa^{2}-\frac{1}{2} \kappa^{4}\right) .
$$

The shot noise vanishes when $\kappa \rightarrow 1$; it is fully due to the charge renormalization.

The Fano factor $F$, the dimensionless ratio of shot-noise power and average current, results as

$$
F=\frac{P_{12}}{e V_{1} G_{12}}=\frac{1}{\kappa^{2}}-\frac{1}{2}\left(1+\kappa^{2}\right) .
$$

\section{Thermoelectricity}

Because of the energy dependence of the effective charge, a temperature difference $\delta T$ between contacts 1 and 2 will produce an electrical current $I_{12}=\alpha_{12} \delta T$ in addition to a heat current. The thermoelectric coefficient $\alpha_{12}$ is given by [17]

$$
\alpha_{12}=\frac{\pi^{2}}{3 e} k_{\mathrm{B}}^{2} T_{0} \lim _{E \rightarrow 0} \frac{d}{d E} G_{12}(E) .
$$

Substitution of Eq. (4.6) gives

$$
\begin{aligned}
\alpha_{12} & =-\frac{\pi^{2}}{3 e} k_{\mathrm{B}}^{2} T_{0} G_{12}(0) \frac{\Delta_{0}^{2}}{\left(\beta^{2}-\Delta_{0}^{2}\right)^{3 / 2}} \\
& =-g_{0} e \kappa_{0}^{2} N_{\text {Landau }} \frac{\Delta_{0}^{2}}{\left(\beta^{2}-\Delta_{0}^{2}\right)^{3 / 2}} \\
& =-g_{0} e N_{\text {Landau }} \frac{\left(\Delta_{0} / \beta\right)^{2}}{\left(\beta^{2}-\Delta_{0}^{2}\right)^{1 / 2}} .
\end{aligned}
$$

\section{NUMERICAL SIMULATIONS}

To test these analytical results, we have carried out numerical calculations in a tight-binding model of the Weyl superconductor with a vortex lattice.

\section{A. Tight-binding Hamiltonian}

The Bogoliubov-de Gennes Hamiltonian $H_{\mathrm{S}}$ in the superconducting region $0<z<L$ is

$$
\begin{aligned}
H_{\mathrm{S}}= & \left(\begin{array}{cc}
H_{0}(\boldsymbol{k}+e \boldsymbol{A}) & \Delta \\
\Delta^{*} & -\sigma_{y} H_{0}^{*}(-\boldsymbol{k}+e \boldsymbol{A}) \sigma_{y}
\end{array}\right), \\
H_{0}(\boldsymbol{k})= & t_{0} \sum_{\alpha=x, y, z}\left[\tau_{z} \sigma_{\alpha} \sin k_{\alpha} a_{0}+\tau_{x} \sigma_{0}\left(1-\cos k_{\alpha} a_{0}\right)\right] \\
& +\beta \tau_{0} \sigma_{z}-\mu \tau_{0} \sigma_{0} .
\end{aligned}
$$

The cubic lattice constant of the tight-binding model is $a_{0}$ and $t_{0}$ is the nearest-neighbor hopping energy. In what follows we will set $a_{0}$ and $t_{0}$ both equal to unity.

In the strong-type-II limit the magnetic field $\boldsymbol{B}=B_{0} \hat{z}$ penetrates the superconductor uniformly, with vector potential $\boldsymbol{A}=\left(-B_{0} y, 0,0\right)$. The absolute value $\Delta_{0}$ of the pair potential $\Delta=\Delta_{0} e^{i \phi}$ can also be taken uniform, assuming that the size $\xi_{0}=\hbar v_{\mathrm{F}} / \Delta_{0}$ of the vortex core is small compared to the magnetic length $l_{m}=\sqrt{\hbar / e B_{0}}$. For the analytical calculations this is the only requirement. For the numerics we also take $\xi_{0}$ small compared to the tight-binding discretization length $a_{0}$, and then ensure that a vortex core (where the phase field is undefined) does not coincide with a lattice point. This implies that $a_{0}$ is large compared to the atomic lattice constant (which itself must be much smaller than $\xi_{0}$ ).

The vortices are arranged on a square lattice in the $x-y$ plane, lattice constant $d_{0}=N_{0} a_{0}$, with two $h / 2 e$ vortices in a unit cell. The number

$$
N_{0}=\left(a_{0}^{2} e B_{0} / h\right)^{-1 / 2}
$$




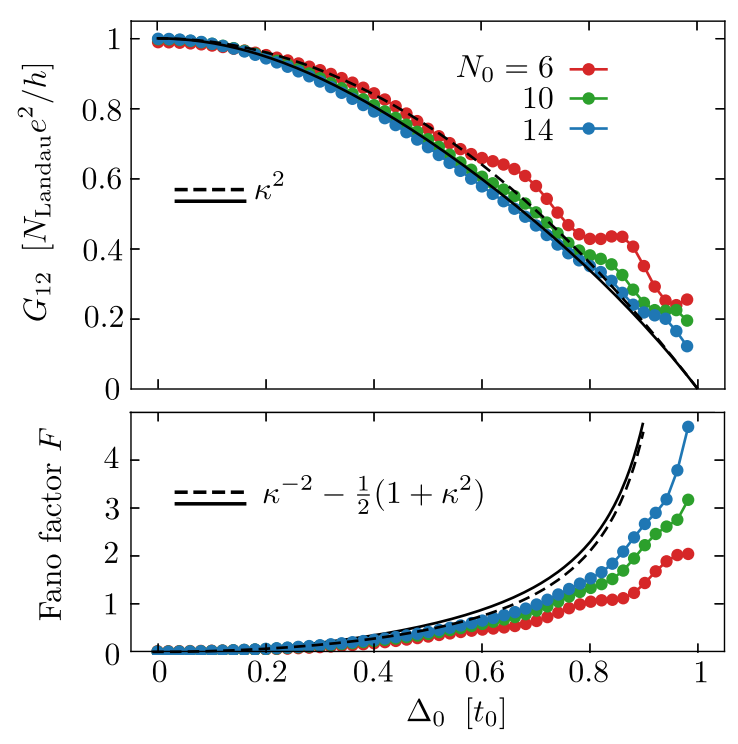

FIG. 4. Data points: Electrical conductance (top) and Fano factor (bottom) in the superconducting vortex lattice (lattice constant $d_{0}$ ), as a function of the pair potential $\Delta_{0}$ at fixed magnetization $\beta=1$, calculated from the tight-binding model (lattice constant $a_{0}$ ) for different lattice constant ratios $N_{0}=d_{0} / a_{0}$. The black curves are the analytical predictions from the charge renormalization factor $\kappa$, both in the approximation of a linearized dispersion (black dashed curve, $\kappa=\kappa_{0}=\sqrt{1-\Delta_{0}^{2} / \beta^{2}}$ ) and for the full nonlinear dispersion (black solid).

is set at an integer value. The phase $\phi(\boldsymbol{r})$ winds around the vortex cores $\boldsymbol{R}_{n}$ according to

$$
\nabla \times \nabla \phi=2 \pi \hat{z} \sum_{n} \delta\left(\boldsymbol{r}-\boldsymbol{R}_{n}\right) .
$$

In the normal-metal leads $z<0, z>L$ we have $\Delta_{0} \equiv 0$ and a large chemical potential $\mu_{\mathrm{N}}$, so only modes with a large longitudinal momentum $k_{z}$ couple to the superconductor. We effectuate the $\mu_{\mathrm{N}} \rightarrow \infty$ limit by removing the transverse $x, y$ couplings in the leads, resulting in the Hamiltonian [18]

$$
H_{\mathrm{N}}=v_{z} \tau_{z} \sigma_{z} \sin k_{z}+v_{z} \tau_{x} \sigma_{0}\left(1-\cos k_{z}\right) .
$$

The gauge-invariant discretization of the Hamiltonian (5.1) in the magnetic Brillouin zone is detailed in Ref. [7]. The scattering matrix is calculated using the KWANT code [19].

\section{B. Results}

Results for the conductance and shot noise are shown in Fig. 4 , as a function of $\Delta_{0}$ for $\beta=1, \mu=0$. The analytical predictions (4.4) for the conductance and (4.9) for the Fano factor are given by the black curves. As a check, for these curves we have also calculated the charge renormalization factor $\kappa$ from the full sinusoidal dispersion, without making the small- $\boldsymbol{k}$ expansion of Eq. (2.1) - the difference with $\kappa_{0}=$ $\sqrt{1-\Delta_{0}^{2} / \beta^{2}}$ is small.

To assess finite-size effects in the numerics we show results for different values of the ratio $N_{0}=d_{0} / a_{0}$ of magnetic unit cell and tight-binding unit cell. As expected, the agreement between numerics and analytics improves with increasing

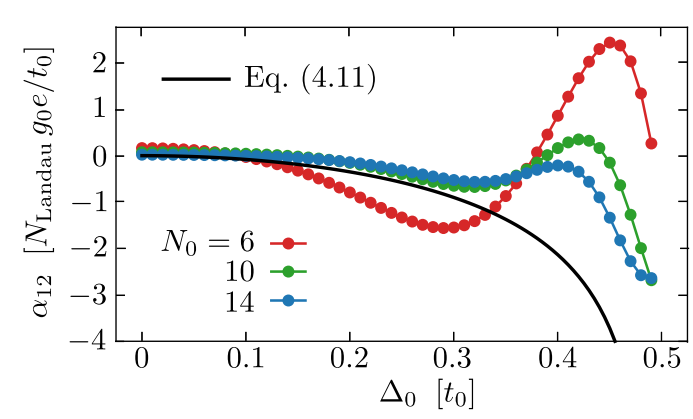

FIG. 5. Dependence on $\Delta_{0}$ for $\beta=0.5$ of the thermoelectric coefficient (4.10), calculated from the infinite-system analytics (black solid curve) or obtained from finite-size numerics (colored data points).

$N_{0}$, for $\Delta_{0} / \beta$ not close to unity. (At $\Delta_{0}=\beta$ the spectrum becomes gapless and the low-energy analytics breaks down.)

These are results at the Fermi level, $E=0$. The energy dependence of the conductance determines the thermoelectric coefficient (4.10). We show numerical results for $\alpha_{12} \propto$ $d G_{12} / d E$ in Fig. 5, for a smaller $\beta=0.5$ to reduce the oscillations that disappear only slowly with increasing $N_{0}$.

\section{Test for isotropy of the charge renormalization}

So far we assumed that the internal magnetization $\beta$ is parallel to the external magnetic field in the $z$ direction. This assumption is needed for our low-energy analytics, but numerically we can take an arbitrary angle between the magnetization $\boldsymbol{\beta}=\left(\beta_{x}, \beta_{y}, \beta_{z}\right)$ and the magnetic field, by replacing the term $\beta \tau_{0} \sigma_{z}$ in the Hamiltonian (5.1b) with $\tau_{0} \boldsymbol{\beta} \cdot \boldsymbol{\sigma}$. Results for $\boldsymbol{\beta}=(\beta, 0,0)$, so for a magnetization perpendicular to the magnetic field, are shown in Fig. 6. There is no qualitative difference with Fig. 4 for the parallel configuration; the quantitative difference is that the finite-size effects are smaller.

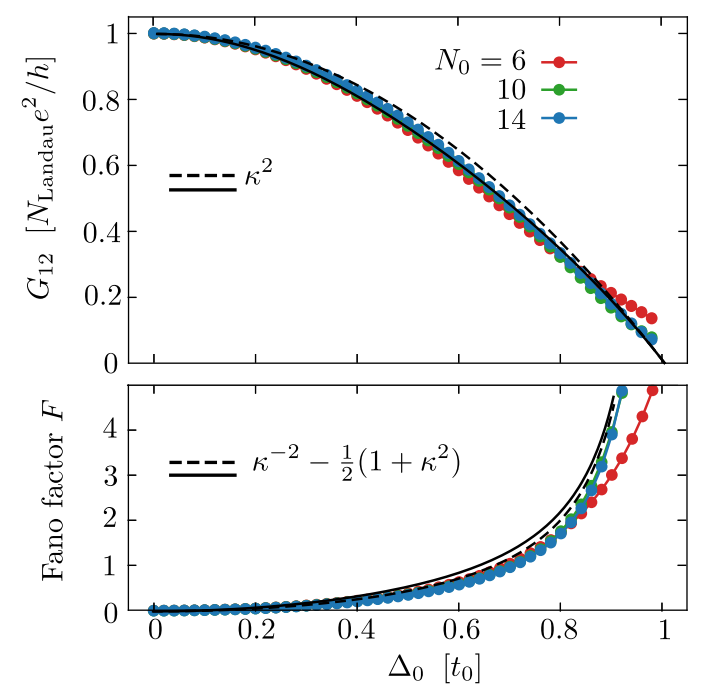

FIG. 6. Same as Fig. 4, but for a magnetization $\beta$ that is perpendicular rather than parallel to the magnetic field $B$. 


\section{CONCLUSION}

In summary, we have shown how the charge renormalization $e \mapsto \kappa e$ of Weyl fermions in a superconducting vortex lattice modifies the electrical and thermoelectrical transport properties.

In the electrical conductance, the current per vortex is reduced by a factor $\frac{1}{2} \kappa^{2}$-a prefactor $1 / 2$ because of the Majorana nature of the quasiparticles and a factor $\kappa^{2}$ because of the effective charge. At the Weyl point $\kappa \rightarrow \kappa_{0}=\sqrt{1-\Delta_{0}^{2} / \beta^{2}}$ depends on the ratio of the superconducting gap $\Delta_{0}$ and the separation $2 \beta$ of the Weyl points of opposite chirality.

The charge-squared renormalization of the electrical conductance is a simple result, but perhaps not what one might have guessed by analogy with the fractional quantum Hall effect, where a 1/3 fractional charge reduces the conductance by $1 / 3$ rather than $1 / 9$. The key difference is that here the quasiparticles are not in an eigenstate of charge; the charge renormalization is due to quantum fluctuations, which give uncorrelated reductions by $\kappa \times \kappa$ at entrance and exit. These quantum fluctuations of the charge are also responsible for the large shot-noise power that we have found, with a diverging Fano factor (4.9) in the limit $\kappa \rightarrow 0$.

The energy dependence of the charge renormalization implies that charge transport parallel to the magnetic field $B$ goes hand-in-hand with heat transport. As a result, a nonzero thermoelectric coefficient $\alpha_{12}$ along the field lines appears in a chiral Landau level—something that would not be possible in the normal state: The Landau level contributes an energyindependent number of propagating modes along $B$ (one mode per flux quantum) and the chirality suppresses backscattering, so the energy derivative in Eq. (4.10) would vanish in the normal state.

There is much recent interest in thermoelectricity of Weyl fermions in a Landau level [20-23], but that refers to currents perpendicular to $B$. Our findings show that charge renormalization in a Weyl superconductor provides a mechanism for a nonzero effect parallel to the field lines.

In our calculations we have assumed a clean system, without impurity scattering. However, we expect the transport properties to be robust against nonmagnetic disorder, which in the effective low-energy Hamiltonian (2.5) would enter as a term proportional to $\sigma_{z}$ that does not couple Landau levels of opposite chirality.

\section{ACKNOWLEDGMENTS}

This project has received funding from the Netherlands Organization for Scientific Research (NWO/OCW), from TÜBİTAK Grant No. 114F163, and from the European Research Council (ERC) under the European Union's Horizon 2020 Framework Programme for Research and Innovation.
[1] T. Meng and L. Balents, Weyl superconductors, Phys. Rev. B 86, 054504 (2012); Erratum: Weyl superconductors [Phys. Rev. B 86, 054504 (2012)], 96, 019901(E) (2017).

[2] A. P. Schnyder and P. M. R. Brydon, Topological surface states in nodal superconductors, J. Phys.: Condens. Matter 27, 243201 (2015).

[3] D. J. Van Harlingen, Phase-sensitive tests of the symmetry of the pairing state in the high-temperature superconductors, Rev. Mod. Phys. 67, 515 (1995).

[4] H. B. Nielsen and M. Ninomiya, The Adler-Bell-Jackiw anomaly and Weyl fermions in a crystal, Phys. Lett. B 130, 389 (1983).

[5] A. M. Turner and A. Vishwanath, Beyond band insulators: Topology of semi-metals and interacting phases, arXiv:1301.0330.

[6] M. Franz and Z. Tešanović, Quasiparticles in the Vortex lattice of Unconventional Superconductors: Bloch Waves or Landau Levels? Phys. Rev. Lett. 84, 554 (2000).

[7] M. J. Pacholski, C. W. J. Beenakker, and I. Adagideli, Topologically Protected Landau Level in the Vortex Lattice of a Weyl Superconductor, Phys. Rev. Lett. 121, 037701 (2018).

[8] C. W. J. Beenakker, Annihilation of Colliding Bogoliubov Quasiparticles Reveals their Majorana Nature, Phys. Rev. Lett. 112, 070604 (2014).

[9] S. R. Elliott and M. Franz, Majorana fermions in nuclear, particle, and solid-state physics, Rev. Mod. Phys. 87, 137 (2015).

[10] P. Baireuther, J. Tworzydło, M. Breitkreiz, I. Adagideli, and C. W. J. Beenakker, Weyl-Majorana solenoid, New J. Phys. 19, 025006 (2017).
[11] Y. Aharonov and A. Casher, Ground state of a spin-1/2 charged particle in a two-dimensional magnetic field, Phys. Rev. A 19, 2461 (1979).

[12] M. I. Katsnelson and M. F. Prokhorova, Zero-energy states in corrugated bilayer graphene, Phys. Rev. B 77, 205424 (2008).

[13] J. Kailasvuori, Pedestrian index theorem à la Aharonov-Casher for bulk threshold modes in corrugated multilayer graphene, Europhys. Lett. 87, 47008 (2009).

[14] G. E. Volovik, Fermion zero modes on vortices in chiral superconductors, JETP Lett. 70, 609 (1999).

[15] L. Fu and C. L. Kane, Superconducting Proximity Effect and Majorana Fermions at the Surface of a Topological Insulator, Phys. Rev. Lett. 100, 096407 (2008).

[16] M. P. Anantram and S. Datta, Current fluctuations in mesoscopic systems with Andreev scattering, Phys. Rev. B 53, 16390 (1996).

[17] U. Sivan and Y. Imry, Multichannel Landauer formula for thermoelectric transport with application to thermopower near the mobility edge, Phys. Rev. B 33, 551 (1986).

[18] We have checked that the continuum limit of the tight-binding Hamiltonian of Sec. V A gives the same analytical results for the transport coefficients as calculated in Sec. IV; see Supplemental Material at http://link.aps.org/supplemental/10.1103/ PhysRevB.100.035417 for an analytic calculation consistent with microscopic model of the junction.

[19] C. W. Groth, M. Wimmer, A. R. Akhmerov, and X. Waintal, Kwant: A software package for quantum transport, New J. Phys. 16, 063065 (2014). 
[20] B. Skinner and L. Fu, Large, nonsaturating thermopower in a quantizing magnetic field, Sci. Adv. 4, eaat2621 (2018).

[21] V. Kozii, B. Skinner, and L. Fu, Thermoelectric Hall conductivity and figure of merit in Dirac/Weyl materials, Phys. Rev. B 99, 155123 (2019).

[22] W. Zhang, P. Wang, B. Skinner, R. Bi, V. Kozii, C.-W. Cho, R. Zhong, J. Schneeloch, D. Yu, G. Gu, L. Fu, X. Wu, and
L. Zhang, Quantized plateau in the thermoelectric Hall conductivity for Dirac electrons in the extreme quantum limit, arXiv:1904.02157.

[23] F. Han, N. Andrejevic, T. Nguyen, V. Kozii, Q. Nguyen, Z. Ding, R. Pablo-Pedro, S. Parjan, B. Skinner, A. Alatas, E. Alp, S. Chi, J. Fernandez-Baca, S. Huang, L. Fu, and M. Li, Discovery of Giant, Non-saturating Thermopower in Topological Semimetal at Quantum Limit, arXiv:1904.03179. 